\title{
La natura organica e l'uomo nel pensiero di Plessner Vallori Rasini
}

I.

Tutte le questioni a cui Helmuth Plessner si è dedicato convergono verso un tema centrale: l'uomo. $^{1}$ All'essere umano, alle condizioni e alle modalità della sua esistenza, alla diversità delle sue concrete manifestazioni individuali e sociali, ha dedicato una lunga vita di brillante pensatore. Del tutto a ragione dunque è considerato uno dei massimi rappresentanti dell' «antropologia filosofica» novecentesca, e I gradi dell'organico e l'uomo è l'opera fondamentale in cui si radica la sua intera concezione. Va comunque precisato che lo snodarsi della riflessione di Plessner oltrepassa senz'altro l'ambito specificamente antropologico, delineando l'immagine di un filosofo profondo e appassionato quanto versatile ed eclettico. ${ }^{2} \mathrm{Nel}$ suo ricco percorso intellettuale, l'interesse per le scienze incontra la passione per la filosofia e la mol-

${ }^{1}$ I principali scritti di Helmuth Plessner sono contenuti nei ro volumi delle Gesammelte Schriften, a cura di G. Dux, O. Marquard ed E. Ströker, Suhrkamp, Frankfurt a. M. 1980-85. Recentemente S. Giammusso e H.-U. Lessing hanno curato una raccolta di saggi non inclusi nelle Gesammelte Schriften: H. Plessner, Politik, Antbropologie, Pbilosophie. Aufsätze und Vorträge, Fink, München 2001. Per la bibliografia degli scritti di Plessner cfr. S. Giammusso, Bibliographie Helmuth Plessners, in «Dilthey Jahrbuch für Philosophie und Geschichte der Geisteswissenschaften», VII, 1990-9I, pp. 323-4I.

${ }^{2}$ Già da tempo, in Germania, la critica rivolge a Plessner - e in particolare a questo suo eccezionale scritto - una più che meritata attenzione, continuando ad arricchire il dibattito sul suo contributo alla filosofia contemporanea. Tra gli studi più recenti - davvero numerosi - ci limitiamo a segnalare: J. Beaufort, Die gesellschaftliche Konstitution der Natur. Helmuth Plessners kritisch-phänomenologische Grundlegung einer bermeneutischen Naturpbilosophie in «Die Stufen des Organischen und der Mensch", Königshausen und Neumann, Würzburg 2000; H. Kämpf, Helmutb Plessner. Eine Einfübrung, Parerga, Düsseldorf 200r; W. Essbach, J. Fischer e H. Lethen 
teplicità degli stimoli culturali di inizio secolo si combina proficuamente con una rara capacità di confrontarsi con il pensiero contemporaneo senza mai smarrire l'autonomia di una posizione critica. Perciò, se rimangono assolutamente decisivi l'approfondimento della filosofia kantiana, l'incontro con la fenomenologia o il fascino per l'ermeneutica storicista, l'originalità delle sue elaborazioni teoriche impedisce qualunque affiliazione di Plessner a un orientamento o a una corrente preesistente. ${ }^{3}$

È caratteristica di Plessner una netta propensione alla completezza, a indagare in ogni direzione e «a tutto campo»: non ritiene che la conoscenza viaggi su un solo binario; meno che mai il sapere filosofico. E quando al centro della ricerca si colloca l'uomo - un oggetto così complesso e sfuggente da restare l'enigma maggiore per se stesso - il ricorso a una indagine polivalente sembra assolutamente inevitabile. Metodi differenti e prospettive complementari devono collaborare in vista di un unico scopo; e solo la filosofia può aspirare a una sintesi unitaria dei vari percorsi, a una composizione - possibilmente sistematica - delle diverse «immagini» dell'uomo. È essenziale infatti considerare l'intera gamma delle possibilità esistenziali dell'essere umano spiegando quali siano le condizioni che gli consentano le manifestazioni più caratte-

(a cura di), Plessners « Grenzen der Gemeinschaft». Eine Debatte, Suhrkamp, Frankfurt a. M. 2002; $\mathrm{K}$. Hauke, Das liberale Ethos der Würde. Eine systematisch orientierte Problemgeschichte zu Helmuth Plessners Begriff menschliche Würde in den "Grenzen der Gemeinschaft», Könighausen und Neumann, Würzburg 2003 (pubblicato dopo un' introduzione a Plessner concentrata principalmente sul significato di Die Stufen des Organischen und der Menscb: Plessner zur Einfübrung, JuniusVerlag, Hamburg 200o); H. H. Holz, Mensch - Natur. Helmuth Plessner und das Konzept einer dialektischen Anthropologie, Transcript, Bielefeld 2003; C. Dejung, Helmuth Plessner. Ein deutscher Philosoph zwischen Kaiserreich und Bonner Republik, Rüffer und Rub, Zürich 2003; Id., Helmuth Plessner interkulturell gelesen, Bautz, Nordhausen 2005. Anche in Italia la notorietà di Plessner comincia ormai a essere considerevole, soprattutto grazie alla traduzione di alcuni suoi rilevanti lavori. Di recente infatti sono stati pubblicati diversi suoi saggi brevi, ma anche opere più consistenti, come Il riso e il pianto. Una ricerca sui limiti del comportamento umano, a cura di V. Rasini, Bompiani, Milano 200o; I limiti della comunità. Per una critica del radicalismo sociale, a cura di B. Accarino, Laterza, Roma-Bari 2001, e Potere e natura umana, a cura di B. Accarino, manifestolibri, Roma 2006; mentre è in corso di traduzione Mit anderen Augen. Aspekte einer philosophischen Antbropologie. Per quanto riguarda gli studi sul filosofo sono da menzionare M. Russo, La provincia dell'uomo. Studio su Helmuth Plessner e sul problema di un'antropologia filosofica, La città del sole, Napoli 2000; O. Tolone, Homo absconditus. L'antropologia filosofica di Helmuth Plessner, ESI, Napoli 2000; V. Rasini, Teorie della realtà organica. Helmuth Plessner e Viktor von Weizsäcker, SIGEM, Modena 2002. Non mancano, infine, saggi brevi su aspetti diversi del suo pensiero apparsi in riviste e volumi collettanei.

${ }^{3} \mathrm{Cfr}$. la Cronologia alle pagine xxv-xxvI. 
ristiche. «Questa questione si può svolgere in due direzioni - chiarisce Plessner - orizzontalmente, cioè nella direzione che è fissata dalla relazione che l'uomo nel suo agire e subire ricerca con il mondo, e verticalmente, cioè nella direzione risultante dalla posizione nel mondo come organismo nella categoria degli organismi, che l'uomo ha sviluppato naturalmente. In entrambe queste direzioni si può sperare di comprendere veramente l'uomo come soggetto-oggetto della cultura e come soggetto-oggetto della natura, senza dividerlo in astrazioni artificiose». ${ }^{4}$ Le due direzioni dello schema di ricerca corrispondono alla distinzione (ingenua e tuttavia reale) dell'appartenenza dell'uomo a due dimensioni: alla dimensione culturale, nella quale si sviluppano le sue facoltà intellettuali e artistiche, si concretizza la propensione al vivere sociale e si rivela la sua storicità; e alla dimensione naturale, secondo la quale l'uomo è un ente biologico facente parte, non meno delle piante e degli animali, del complesso sistema dei viventi. Questa sua doppia appartenenza crea continue intersezioni, un incrociarsi di piani esistenziali che mette l'uomo in conflitto con se stesso. Ma questa è la sua essenza, e solo percorrendone fino in fondo la duplicità sarà possibile trovare la radice, il fondamento unitario che le dà origine. Per questo le due direzioni dell'indagine divergono solo in apparenza: di fatto si implicano reciprocamente, conducendo a conclusioni comuni.

A un'opera precedente, Die Einheit der Sinne, pubblicata nel 1923 , Plessner aveva affidato alcune indagini svolte nella prima direzione.5 I gradi dell'organico e l'uomo ha invece il compito di avviare il percorso «verticale» della ricerca sulla «totalità» dell'uomo. Ma non sarà solo così: l'opera guadagnerà un ruolo ben più decisivo. Le spetterà infatti il lavoro di fondazione a priori della realtà psicofisica umana, ma prima ancora quella dell'essere organico in generale, e da un punto di vista metodologico la «filosofia della natura» che ne risulterà finirà per collocarsi anche alla base di ogni indagine sulla dimensione spirituale: «Senza una filosofia dell'uomo - dichiara infatti espressamente Plessner - nessuna teoria dell'esperienza umana della vita nelle scienze dello spirito. Senza una filosofia della natura, nessuna filosofia dell'uomo». ${ }^{6}$

\footnotetext{
${ }^{4}$ Infra, p. 56.

${ }^{5}$ H. Plessner, Die Einheit der Sinne. Grundlinien einer Ästesiologie des Geistes (1923), in Gesammelte Schriften cit., vol. III, 1980, pp. 7-315.

${ }^{6}$ Infra, p. 50.
} 
I gradi dell'organico e l'uomo deve individuare la posizione che contraddistingue l'essere umano nel generale progetto della natura e definirne similarità e differenze rispetto alle altre forme del vivente. $\mathrm{Ne}$ deriva anzitutto un «primato» della corporeità: l'essere umano è corpo, un ente in carne e ossa capace di sentire e di agire, legato a un'esistenza concreta le cui specificità dipendono precisamente dalla sua costituzione fisica. Lo studio della configurazione organica diviene essenziale e prioritaria, fondante per ogni indagine antropologica, qualunque ne sia la direzione. Ma risulta altresì che una «filosofia della natura», la quale - precisa Plessner - va intesa «nel suo senso più ampio e originario», ${ }^{7}$ assolve in un certo senso la funzione di una «filosofia prima».

Si tratterà di una teoria che sviluppa le principali questioni legate all'aspetto materiale e sensibile del vivente in una forma «non empiricamente ristretta ${ }^{8}{ }^{8}$ vale a dire in una forma filosofica non limitata alle modalità metodologiche e concettuali utilizzate dalla scienza. Non che la scienza proceda in modo errato: al contrario, le indagini scientifiche ottengono risultati indiscutibilmente significativi, e il riconoscimento di Plessner è pieno. Ma la strada sulla quale procede la scienza è diversa da quella della filosofia: una «filosofia della natura» non si sovrappone e non si sostituisce alla teoria scientifica, perché si colloca su un altro piano, sonda altri percorsi possibili, si avvale di altri strumenti; e l'esperienza che interroga ha una valenza in qualche modo «fenomenologica», non semplicemente empirica, che copre un campo più esteso. ${ }^{9}$ Il suo appello ai sensi e all'intuizione travalica dunque le restrizioni della rappresentabilità fisico-matematica.

2.

I gradi dell'organico e l'uomo uscì nel 1928 , in un momento di grande interesse per le questioni antropologiche e, più in generale, in un frangente storico ricco di fermento sia in ambito filosofico sia in ambito scientifico. Benché si possa pensare, considerando la mole e la siste-

${ }^{7}$ Infra, p. 48.

${ }^{8}$ Infra, p. 50.

${ }^{9}$ Sul peculiare rapporto di Plessner con la fenomenologia mi permetto di rimandare a V. Rasini, Il pensiero fenomenologico secondo Plessner, in «Annali del Dipartimento di Filosofia di Firenze», 2003-04, pp. 267-80. 
maticità dell'opera, a un lavoro di stesura decisamente rapida, ${ }^{10} \mathrm{I}$ gradi dell'organico e l'uomo era di fatto in laboratorio da qualche tempo. Già nel I924, ne era stata annunciata l'imminente pubblicazione come secondo volume del saggio di «estesiologia dello spirito» apparso l'anno precedente. L'anticipazione proponeva il titolo Pflanze, Tier, Mensch. Elemente einer Kosmologie der lebendigen Form. ${ }^{11} \mathrm{Ma}$ furono necessari alcuni anni prima che il lavoro fosse ultimato e, quando apparve, il progetto all'interno del quale questa ricerca veniva a collocarsi si era almeno in parte trasformato. Die Einheit der Sinne si muoveva nel contesto di una teoria della conoscenza, più precisamente di una teoria della sensibilità e di una «critica dei sensi». ${ }^{12}$ Per completare la ricerca sulle modalità dei processi percettivi, per raggiungere insomma la chiave della «unità» dei sensi, si rendeva necessario sviluppare anche una teoria della struttura dell'essere umano e delle possibilità collegate a tale struttura.

Così - ci racconta Plessner - è stato grazie agli interessi estesiologici e alle ricerche condotte nell'opera del I 923 che si poté aprire «il varco verso l'antropologia filosofica».$^{13} \mathrm{Ma}$ l'opera che si produsse attraverso questo varco, come abbiamo detto, venne maturando una sua profonda autonomia. Non si trattò più, infatti, di fornire puntelli di sostegno a una teoria della conoscenza, ma di indagare gli strati più profondi della struttura organica, di definire la natura del vivente caratterizzandone l' «essere» rispetto alla natura inanimata. Una indagine «ontologica», quindi. Ma il termine non va equivocato. Plessner non ha intenzione di concedere spazio alla metafisica: l'indagine sull'essere del vivente va intesa nel suo significato più generale di «analisi dei caratteri essenziali» di un certo tipo di ente, vale a dire come determinazione a priori degli elementi specifici che lo rendono tale. Richiamandosi a Helmholtz, Plessner ha voluto denominarla «teoria dei modali organici». Si tratta di una deduzione delle «qualità ultime» del vivente, cioè di quelle qualità che non possono essere ridotte ad altre

${ }^{10} \mathrm{Cfr}$. Dejung, Helmuth Plessner cit., p. 217.

${ }^{11} \mathrm{Si}$ tratta della prefazione a H. Plessner, Grenzen der Gemeinschaft. Eine Kritik des sozialen Radikalismus (1924), in Gesammelte Schriften cit., vol. V, 1981, pp. 7-133; trad. it. I limiti della comunità cit., p. 5. Cfr. inoltre infra, p. 3, dove Plessner sostiene di averne «concepito il progetto" già durante la stesura dell'opera del 1923.

${ }^{12}$ Id., Die Einheit der Sinne cit., p. 76.

${ }^{13}$ Id., Mit anderen Augen. Aspekte einer philosophischen Anthropologie, Reclam, Stuttgart 1982, p. 5; Id., Selbstdarstellung, in Gesammelte Schriften cit., vol. X, 1985, p. 322. 
qualità e che definiscono inequivocabilmente la vita e le modalità dell'organico. ${ }^{14}$

Trovare le qualità essenziali del vivente è il gradino preliminare per una filosofia della natura. Non può esserci una qualunque teoria del vivente senza una definizione della vita, senza una identificazione precisa delle caratteristiche e delle proprietà che fanno di un essere concreto un organismo. Ma come risalire a queste proprietà, in che modo determinarle e soprattutto con quali strumenti? L'esperienza quotidiana ci dà sicuramente un punto di partenza: ci consente di capire - con buone probabilità di essere nel giusto - se ci troviamo dinanzi a un corpo vivente oppure a un corpo inanimato. Una prima indicazione proviene così dalla percezione, dalla constatazione di un certo tipico movimento che sappiamo accompagnare in genere la vita. $\mathrm{Ma}$ talvolta ci inganniamo: quel movimento è solo apparente, è prodotto con l'artificio e non appartiene essenzialmente all'ente che stiamo osservando. $\mathrm{Ci}$ sono tuttavia movimenti che "per essenza» appartengono a un certo ente e solo per errore vengono attribuiti al semplice corpo fisico. Meglio ancora: ci sono corpi che «devono» possedere un certo tipo di movimento, perché la loro struttura costitutiva è tale da non poterlo evitare. Questo speciale movimento - la vitalità - verrà precisato secondo un principio a priori, in termini logico-dialettici, e costituirà il requisito fondamentale in base al quale saranno definite le ulteriori caratteristiche del vivente e le sue diverse forme.

Che per Plessner questa teoria a priori dei caratteri d'essenza organici, nel suo significato e nelle sue acquisizioni fondamentali, conservi intatta negli anni la propria validità è provato dal fatto che nel 1965 , a quasi quarant'anni dalla prima edizione, I gradi dell'organico e l'uomo venga ripresentato alle stampe senza cambiamenti. ${ }^{15}$ Certo - riconosce l'autore nella Premessa alla seconda edizione - l'esposizione può forse apparire segnata dal tempo, ma i contenuti mantengono inalterata la loro pregnanza, e in particolare il concetto di «posizionalità» viene riconosciuto capace di descrivere nel migliore dei modi le peculiarità del vivente, evitando il ricorso a insufficienti concetti psicologici. ${ }^{16}$

${ }^{14}$ Infra, p. 134.

${ }^{15}$ L'edizione del 1965 , pubblicata (come la prima) dall'editore W. de Gruyter, differisce da quella del r 928 per l'aggiunta di una Premessa alla seconda edizione (cfr. infra, pp. 7-25) e di un'Appendice (cfr. infra, pp. $369-83$ ), nella quale Plessner si limita a fornire alcuni chiarimenti e rettifiche.

${ }^{16}$ Infra, p. 20 
Ancora nel 1982, in apertura alla raccolta di saggi dal titolo Mit anderen Augen, Plessner richiama alcune delle tesi fondamentali sviluppate nei Gradi dell'organico e l'uomo e ne riprende alla lettera parte del settimo capitolo sotto il titolo Der Mensch als Lebewesen. ${ }^{17} \mathrm{La}$ scelta ha una precisa motivazione: in maniera teoreticamente rigorosa e convincente, l'opera del ' 28 tentava di rispondere alla domanda sulle condizioni di possibilità dell'essere umano segnando un nuovo inizio per l'antropologia filosofica attraverso un'impostazione dell'indagine che, facendo leva sul rapporto del corpo con il proprio limite, offriva all'organismo in generale e a quello umano in particolare lo statuto della totalità. Abbandonava dunque la via dello «sdoppiamento» dell'uomo in interiorità ed esteriorità, spirito e materia e lo considerava una "unità organica». Questa impostazione toglieva finalmente dall'imbarazzo di fare dell'uomo un «misterioso conjunctum», per restituirlo al regno della vita. ${ }^{18}$

3.

Dal punto di vista filosofico, il vivente deve essere studiato nelle sue diverse proprietà e forme mediante un principio di continuità. Perciò anche l'uomo, nonostante le sue peculiarità, deve rendersi accessibile alla medesima chiave interpretativa valida per il restante mondo organico. L'uomo è un vivente tra viventi, e precisamente a partire da questo dato di fatto si deve poter individuare il motivo della sua evidente «doppia appartenenza». "Nessuno mette in dubbio la straordinaria utilità pratica e la chiarezza della distinzione tra fisico $\mathrm{e}$ psichico. Sicuramente essa coglie differenze essenziali nell'essere della realtà, come attesta il progresso delle scienze del corpo e di quelle della psiche. Ma considerarla un fondamento incontra ormai dubbi e opposizioni non più solo da parte dei filosofi, ma anche da parte di quegli scienziati empirici che hanno a che fare con i misteriosi legami tra fisico e psichico nel formarsi della persona e delle sue prestazio-

${ }^{17} \mathrm{Cfr}$. Plessner, Mit anderen Augen cit., pp. 9-62. Il saggio non va confuso con un altro scritto dal medesimo titolo, tradotto in italiano (L'uomo come essere biologico) nella raccolta di A. Babolin (a cura di), Filosofi tedeschi d'oggi, il Mulino, Bologna 1967, pp. 355-76. Per le parti riprese da Die Stufen des Organischen und der Mensch, cfr. in particolare infra, pp. 332 sgg.

${ }^{18}$ Plessner, Mit anderen Augen cit., p. 6. 
ni». ${ }^{19}$ Non restava dunque che abbandonare l'idea di una «scissione preliminare» della natura umana e proporre alternative più convincenti.

Quella di Plessner - tanto originale quanto impegnativa - non può non suscitare interesse e ammirazione. Eppure, al momento della sua pubblicazione, I gradi dell'organico e l'uomo rimase nell'ombra, priva di attenzione e incapace di suscitare un vero dibattito. I motivi dell'insuccesso furono sicuramente molti, e non mancarono - a quanto pare - valutazioni affrettate ed equivoci ingiustificati, come quello che scambiava la deduzione dei modali organici per un rimaneggiamento filosofico - semplicistico e ingenuo - della teoria dell'evoluzione. ${ }^{20}$ Un lavoro filosofico non può e non deve confondersi con una teoria scientifica: «spetta alla filogenesi - spiega chiaramente Plessner - stabilire se le forme animali provengano da quelle vegetali o se l'uomo si sia sviluppato dall'animale»; la ricerca filosofica ha un altro scopo: si chiede se mai «che cosa significhi dal punto di vista logico una simile graduazione», ${ }^{21}$ ma non entra nel merito della validità dei costrutti teorici della scienza. La «filosofia della natura» non ha a che fare con dati empirici attestanti le modalità di trasformazione degli organismi e la loro maggiore o minore complessità, ma con una «logica della forma vivente», con una interpretazione filosofica (benché non per questo meno rigorosa della spiegazione scientifica) di quali siano le condizioni di possibilità dell'ente organico e dell'uomo.

Ancora più pesantemente, almeno agli occhi di Plessner, gravò il sospetto che l'opera fosse solo una «arringa in favore dell'ilozoismo», una costruzione filosofica che rinnovando antiche concezioni attribuiva la vita alla materia in quanto tale, dissolvendo di fatto la distinzione tra vivente e non vivente. ${ }^{22} \mathrm{Ma}$ appurare la veridicità di una simile ipotesi, di un avvicinamento e un'assimilazione della materia inorganica all'organica - non è compito della filosofia, bensì, se mai, della biochimica. E in ogni caso, l'opera si proponeva precisamente il

\footnotetext{
${ }^{19}$ Infra, p. 63.

${ }^{20}$ E lo stesso Plessner a lamentare questa circostanza. Si veda la premura con cui in, più luoghi, insiste nel dire che «I gradi dell'organico e l'uomo non intende essere un'abbreviazione della teoria evoluzionistica» (Plessner, Selbstdarstellung cit., p. 327; Id., Mit anderen Augen cit., p. 6).

${ }^{21}$ Id., Selbstdarstellung cit., pp. 324-25.

${ }^{22}$ Ibid., p. 325.
} 
compito di individuare la distinzione tra vivente e non vivente, e non di riconoscere la vita ovunque.

$\mathrm{Ma}$ le difficoltà incontrate dall'opera non furono solo queste. «Per la risonanza che ebbe I gradi dell'organico e l'uomo - sostiene Plessner è stato decisivo che l'anno di pubblicazione, il 1928 , coincidesse con la comparsa in brossura della conferenza di Scheler e di Sein und Zeit di Heidegger». ${ }^{23}$ Quanto al primo ostacolo, il saggio al quale si riferisce Plessner è la versione stampata della celebre conferenza di Darmstadt tenuta da Scheler nell'aprile del 1927. Die Stellung des Menschen im Kosmos, ${ }^{24}$ che si propone come il manifesto di una nuova ricerca antropologica, avanzava le istanze principali alle quali di fatto anche Plessner - più giovane e meno noto - si appellava. Niente di più ovvio, quindi, che pensare a Scheler come a una «matrice originale» a cui allievi e giovani pensatori - privi però di una loro autonomia teoretica - si agganciavano nel difendere posizioni condivise. Il rischio di non apparire originale era dunque per Plessner più che reale. Perfino lo stesso Scheler - docente a Colonia, dove anche Plessner svolgeva la propria attività - lo accusò di plagio, senza comunque conoscere veramente il contenuto della sua opera; pare infatti che Scheler non avesse mai minimamente considerato il suo lavoro. ${ }^{25} \mathrm{E}$ certamente vero che alcuni concetti (ma più spesso solo alcuni termini) utilizzati da Plessner rimandano alle ricerche di Scheler, ma il contesto teorico in cui si collocano e l'impostazione generale dell'opera sono assolutamente indipendenti dal suo pensiero. Quella del plagio fu dunque una congettura del tutto priva di valore, e l'equivoco poté risolversi presto grazie all'intervento di Nicolai Hartmann, che aveva avuto modo di conoscere meglio l'opera di Plessner. Dell'incidente tuttavia rimase traccia: sicuramente nel sottotitolo dell'opera che, per esplicita richiesta di Scheler, venne modificato da «fondazione dell'antropologia filosofica» in un più modesto «introduzione all'antropologia filosofica». ${ }^{26} \mathrm{Ma}$ è ragionevole pensare che in ambito accademico l'episodio non abbia giovato alla fama del lavoro.

${ }^{23}$ Plessner, Selbstdarstellung cit., pp. 328-29.

${ }^{24} \mathrm{M}$. Scheler, Die Stellung des Menschen im Kosmos, in Gesammelte Werke, vol. IX, Francke, Bern I975; trad. it. La posizione dell'uomo nel cosmo, Armando, Roma I997; e La posizione dell'uomo nel cosmo, Angeli, Milano 2000 (dall'edizione originale del 1928).

${ }^{25}$ Cfr. quanto scrive Plessner a questo proposito nella Selbstdarstellung cit., p. 329.

${ }^{26} \mathrm{Ibid}$. 
Per quanto riguarda invece Sein und Zeit, è certo che il suo grande successo e la capacità di coinvolgimento delle tematiche esistenzialistiche sottrassero attenzione al neonato orientamento antropologico in filosofia, tanto più se si considera l'articolarsi contemporaneo di una sfavorevole querelle contro «l'antropologismo» che coinvolse personaggi di grande notorietà come, tra gli altri, Husserl. «La scoperta del concetto di esistenza - spiega Plessner nella Premessa alla seconda edizione dei Gradi dell'organico e l'uomo - sembrava fornire la chiave (decidendo così del destino dell'antropologia filosofica) per la soluzione di quelle difficoltà che le scienze dell'uomo, particolarmente sofferenti per la separazione tra la metodologia delle scienze naturali e quella delle scienze dello spirito, non potevano risolvere: la psicologia e la psicopatologia, come pure le branche della medicina interna gravate da questioni psicosomatiche, l'etnologia, la preistoria e la storia dell'evoluzione dell'uomo». ${ }^{27}$

Non l'indagine antropologica, quindi, si adattava alle esigenze di un'epoca travagliata sia sul piano storico sia sul piano culturale, ma una proposta ontologica «radicale», che tendeva a fare terra bruciata di ogni altra impostazione e tematizzazione filosofica; insomma - dichiara amaramente Plessner - «l'ateismo metodologico di questa distruzione, che non si arresta dinanzi ad alcun consolidamento concettuale nella storia del pensiero occidentale, piacque alla generazione scossa dalla guerra», ${ }^{28}$ che le sacrificò persino il rispetto di un grande pensatore come Scheler. Il giovane Plessner non poteva dunque aspettarsi più di tanto. Anche volendo minimizzare l'incidenza del carattere oggettivamente complesso dell'opera, il momento pareva non favorire affatto la sua proposta. Infine, ebbero la parola decisiva i fatti storici: di padre ebreo, Plessner fu presto costretto all'esilio, e ogni eventuale, possibile valutazione della portata innovativa del suo lavoro dovette cedere alle circostanze. ${ }^{29}$

${ }^{27}$ Infra, p. 8.

${ }^{28}$ Infra, p. Io.

${ }^{29}$ Plessner infatti andò in esilio in Turchia e dopo un anno, grazie all'interessamento di F. J. J. Buytendijk, si trasferì a Groningen, dove rimase a lungo, sino al rientro in Germania. 
4.

Nonostante tutto, l'opera I gradi dell'organico e l'uomo va considerata un lavoro di straordinaria originalità e indiscutibile spessore teorico. $E$ anche se non esente da possibili osservazioni di carattere metodologico e concettuale, già solo il rigore filosofico con cui viene impostata e condotta la deduzione dei modali organici giustifica ben più di un semplice interesse storico nei suoi confronti.

Il concetto di «posizionalità» è alla base della definizione dei diversi aspetti del sistema organico e dei suoi processi di sviluppo, delle varie forme di organizzazione del vivente e della singolare «eccentricità» dell'essere umano. Il momento centrale dell'intuizione che conduce a tale concetto è costituito dal cosiddetto principio di «realizzazione del limite». L'idea di fondo è che se si può pensare il limite (in qualunque senso lo si intenda), questo è già in qualche modo superato. Di limite, dunque, si può parlare nel senso negativo più comune come del confine che conclude l'estensione di un corpo; ma si può parlarne anche in un altro senso, positivo e dalla valenza eminentemente dialettica: come di ciò mediante cui un corpo definisce e al contempo oltrepassa se stesso. Se il limite non è un vuoto «stare in mezzo» tra il corpo e ciò che gli sta accanto, ma è «realizzato», cioè appartiene al corpo effettivamente come sua proprietà, allora il corpo possiede anche il «passaggio» che lo trascende: è «in se stesso» (in quanto delimitato e chiuso nei propri confini) e insieme «oltre se stesso" (cioè proiettato al di là del limite che gli appartiene). ${ }^{30} \mathrm{Un}$ principio logico semplice, che marcherà ogni manifestazione della vita e segnerà profondamente il destino dell'uomo.

«Posizionale» è un corpo che «si oltrepassa» nel suo essere, in quanto plastico, attivo e mutevole, continuamente «altro» rispetto a ciò che appare, e perciò difficilmente definibile tramite le categorie della staticità. Il semplice corpo fisico «è»; l'organismo invece «diviene se stesso». I suoi limiti non determinano la fine del suo essere (se non in quanto ente materiale), ma lo mettono in rapporto con l'esterno e con l'«altro da sé». Per questa ragione il vivente è «posto», cioè - potremmo dire - «in corso d'essere». Per indicare questa particolarità

${ }^{30}$ Cfr. infra, pp. 126 sgg. 
essenziale Plessner sceglie il verbo setzen: «L'espressione "porre" ha il suo senso appropriato solo nel compromesso di tale determinazione, nella misura in cui essa evoca il momento dell'essere sollevato, dell'essere sospeso, senza perdere tuttavia l'altro momento, quello dell'essere in quiete, dell'essere stabile». ${ }^{31}$ Poiché l'essere organico, allo stesso tempo, «è»e «non è ancora» (o «non è più»), non lo si può facilmente definire mediante espressioni che ne fissano lo status. Questo termine è in grado di trasmettere il giusto dinamismo, l'idea di qualcosa a cui male si adattano formule «statiche».

Tutti gli aspetti dell'individuo vivente sono descrivibili mediante questa proprietà. $\mathrm{Ma}$ anche le diverse forme dell'organico, nei suoi gradi di evoluzione e nella sua progressione naturale, si rendono intelligibili attraverso lo sviluppo delle potenzialità «posizionali». $\mathrm{Ne}$ deriva una progressione della natura vivente che, a seconda della maggiore o minore autonomia dell'organismo dall'ambiente e della «distanza» da se stesso, scandisce i regni naturali in una successione che va dal regno vegetale a quello umano. Non si tratta di una graduazione determinata dalla «eccellenza» del grado - vale a dire da un suo «valore»-, ma dal livello di sviluppo del principio rispetto al quale il vivente si definisce.

La pianta appartiene al grado inferiore dell'evoluzione posizionale e si caratterizza come «forma aperta», cioè come un organismo proteso verso l'esterno e scarsamente indipendente dal ciclo vitale in cui è inserito. Proprio questa mancanza di autonomia tradisce la semplicità di un ente privo di un'organizzazione capace di guidarlo in azioni finalizzate e consapevoli. ${ }^{32}$ L'animale appartiene invece alla forma «chiusa» dell'organizzazione vitale. In questo grado dello sviluppo posizionale il vivente gode di una maggiore autonomia. Rappresenta un individuo ben separato dall'ambiente, dotato della facoltà di sentire e di agire, e per lo più evidentemente consapevole della possibilità di utilizzare il proprio corpo nell'intervento sull' «altro». $\mathrm{Ma}$, concentrato sulla propria attualità, l'animale non è ancora veramente in rapporto con se stesso. È consapevole, ma non lo è della propria coscienza; sa e agisce, ma gli manca il sapere concernente questa sua realtà. Per questo Plessner parla di organismo «centrico»: la sua posi-

${ }^{31}$ Infra, p. 156 .

${ }^{32}$ Cfr. infra, p. 243. 
zione, centrata sulla sua individualità autonoma, nón gli consente di «oltrepassarsi», di «uscire da sé» per porsi oltre. ${ }^{33}$

A essere invece profondamente in sé e oltre sé è l'uomo. Il concetto di «eccentricità» esprime esattamente il superamento della posizione tipica dell'animale e la doppia necessità per l'ente umano di essere il più prossimo a se stesso e al contempo il più lontano da sé, al punto da rappresentare il proprio, più autentico «altro». Le tre leggi antropologiche che vengono descritte nel capitolo finale dell'opera presentano le diverse sfumature vitali di questa realtà e raccontano, in estrema sintesi, la travagliata storia dell'esistenza più inquieta. ${ }^{34}$

L'uomo non ha pace: non ha patria, non ha un luogo, non ha certezze assolute. La cerca (è anzi l'unico vivente a farlo), ma costitutivamente, cioè per motivi essenziali, non può trovarla, poiché è sempre in grado di revocare in dubbio qualunque acquisizione del proprio smisurato ingegno. Con il grado organico dell'uomo - dice Plessner «è data la condizione affinché il centro della posizionalità abbia distanza da se stesso e renda possibile, nella distinzione da sé, la totale riflessività del sistema di vita. [...] Sebbene anche in questo grado l'essere vivente sia assorbito nel qui e ora e viva a partire dal centro, gli è divenuta cosciente la centralità della sua esistenza. Ha se stesso, sa di sé, è percettibile a se stesso e per questo è un io, il punto di fuga, collocato "dietro di sé", della propria interiorità, che, sottratto a ogni possibile realizzazione della vita a partire dal proprio centro, forma lo spettatore che sta di fronte allo scenario di questo ambito interiore, il polo soggettivo non più oggettivabile, non più trasferibile nella posizione dell'oggetto. In questo ultimo grado della vita si trova la base per sempre nuovi atti della riflessione su se stessi, per un regressus ad infinitum dell'autocoscienza, e con ciò - conclude Plessner - è compiuta la scissione tra sfera esterna, sfera interiore e coscienza $\gg .{ }^{35}$ Come posto nel limite del proprio essere, l'uomo sperimenta i prodigi della mediazione e vive tutta la drammaticità della scissione. Non può fermarsi; non può quietarsi. Ogni sapere, ogni acquisizione, sarà la molla per un passo ulteriore; e ogni passo ulteriore sarà una carta giocata

${ }^{33}$ Infra, p. 25 I.

${ }^{34} \mathrm{Cfr}$. infra, pp. $332 \mathrm{sgg}$.

${ }^{35}$ Infra, pp. 3 I4-r 5 . 
- inevitabilmente - pro e contro se stesso. Plessner ci conduce quindi a grande distanza dall'immagine artificiale e rassicurante di un uomo «padrone del mondo», trascinandoci al cospetto di una quotidianità incerta che insistentemente reclama l'attribuzione di un senso. 